\title{
FIVE NEW SPECIES OF THE GENUS APANTELES FOERSTER (HYMENOPTERA: BRACONIDAE: MICROGASTRINAE) FROM VIETNAM
}

\author{
KHUAT DANG LONG \\ Institute of Ecology and Biological Resources
}

\begin{abstract}
Five new species of two groups of the genus Apanteles Foerster described and illustrated from Vietnam, of which four species Apanteles derivatus Long sp. n., A. flavicentrus Long, sp. n., A. flavigaster Long, sp. n. and A. hatinhensis Long, sp. n. belong to the Apanteles merula - group, one species Apanteles paraglaope Long, sp. n. belongs to the Apanteles ater - group.

Key words: Braconidae, Microgastrinae, Apanteles, merula - group, ater - group, new species, Vietnam.
\end{abstract}

The genus Apanteles Foerster, 1862 s.l. is large and diverse conglomerate in the subfamily Microgastrinae (Braconidae). The genus Apanteles is well known because of their economic importance, the species of Apanteles are abundant everywhere in terrestrial habitats and frequently reared from larvae of many lepidopterious insects that they are readily recognized by most entomologists. The genus is so diverse that not always easy to identify the species. Nixon (1965) have divided the genus into 44 species - groups and Mason (1981), based on groups divided by Nixon (1965), and named 28 genera. However, of those only some of the genera recognized by Mason (1981) had valid names [8].

Nixon (1965) described and keyed 21 old world species of Apanteles merula - group, of those 5 species from Africa, 7 species from Europe and 9 species from the Indo - Australian region. Kotenko (1981) described one new species and keyed 12 species of Apanteles merula - group, Europe. Khuat Dang Long (2007) described two new species of this group,
A. gialamensis Long K, 2007 and A. subcamilla Long K, 2007, from Vietnam. According to Nixon (1965), the merula - group has the cubitellan cell of the hind wing not longer than wide, but all species of this group from Vietnam has the cubitellan cell longer than wide. Among 125 species of Apanteles ater - group keyed by Nixon (1965), one species has a brownish cloud beneath the pterostigma, A. aglaope, the other one new species with this aberrant character belonging to Apanteles ater - group is described from Vietnam, A. paraglaope sp. n..

The terminology used in this paper follows van Achterberg (1993), description format follows Khuat Dang Long (2007), for the division in groups of the genus Apanteles s.l., see Nixon (1965), for reclassification of the subfamily Microgastrinae and for the recognition of the genera, see Mason (1981). All the drawings were done by the author; the scale lines of the plates indicate $1 \mathrm{~mm}$. All types are deposited in the collection of Department of Insect Ecology at IEBR.

\section{TAXONOMY}

\section{Key to Vietnamese species of the Apanteles merula - group}

1. Ocelli situated in rather high triangle, anterior tangent of posterior ocelli not touching lower margin of anterior ocellus (fig. 2).

- Ocelli situated in low triangle, anterior tangent of posterior ocelli cutting anterior ocellus (figs 6, 12 and 16)......

2. Pterostigma pallid with a darker border; ovipositor nearly straight basally, abruptly curved at apex (fig. 7, Khuat Dang Long, 2007: 26)... ..A. gialamensis Long K, 2007 
- Pterostigma brown; ovipositor evenly curved throughout (fig. 3)

A. derivatus sp. $\mathrm{n}$.

3. First metasomal tergite weakly narrowed at apex (fig. 14 and fig. 12, Khuat Dang Long, 2007: 28).

- First metasomal tergite distinctly narrowed behind middle to apex (figs 9 and 19)

4. Metacarp rather short, 3.0 times as long as distance from it to apex of the marginal cell; pterostigma pallid with a darker border (fig. 13, Khuat Dang Long, 2007: 28); metasoma black

A. subcamilla Long K, 2007

- Metacarp very long, 7.5 times as long as distance from it to apex of the marginal cell (fig. 15); pterostigma entirely brown; metasoma entirely yellow.... A. flavigaster sp. $\mathrm{n}$.

5. Eyes more or less convergent below (fig. 7); hind femur dark brown; ovipositor sheath slightly longer hind tibia; ovipositor weakly curved down apically (fig. 10). A. flavicentrus sp. $\mathrm{n}$.

- Eyes not convergent below (fig. 17); hind femur yellow; ovipositor sheath much longer hind tibia; ovipositor nearly straight basally, abruptly curved at apex (fig. 18)......A. hatinhensis sp. $\mathrm{n}$.

Apanteles gialamenesis Long K, 2007 $1-7$.

TAP CHI SINH HOC, 2007, 29(3): 25, figs

\section{A. subcamilla Long K, 2007}

TAP CHI SINH HOC, 2007, 29(3): 27, figs 8-13.

Apanteles derivatus Long, sp. n. (Figs 1-5)

Material: Holotype, ${ }_{+}$, Apan.863, (IEBR), N.W. Vietnam: Lao Cai, Nam Cuong, secondary forest, 11.vi.2004, K. D. Long.

Holotype: $q$, length of body $2.2 \mathrm{~mm}$, of fore wing $2.6 \mathrm{~mm}$, of setose part of ovipositor sheath $0.7 \mathrm{~mm}$ and of antenna $2.3 \mathrm{~mm}$.
Head: antenna slightly longer than body; eyes weakly convergent below (fig. 1); width of face 0.8 times height of eye (12:15), and 0.85 times length of face and clypeus combined (12:14); distance between tentorial pits 3.5 times distance from pit to eye margin. Head in dorsal view transverse, its width 1.9 times median length; ocelli in high triangle, anterior tangent of posterior ocelli not touching posterior margin of anterior ocellus; distance between posterior ocelli (POL) 1.75 times diameter of posterior ocellus (OD) and 0.7 times distance from posterior ocellus to eye margin (OOL) (fig. 2). Face, frons, vertex and temple shiny with sparse fine punctures.

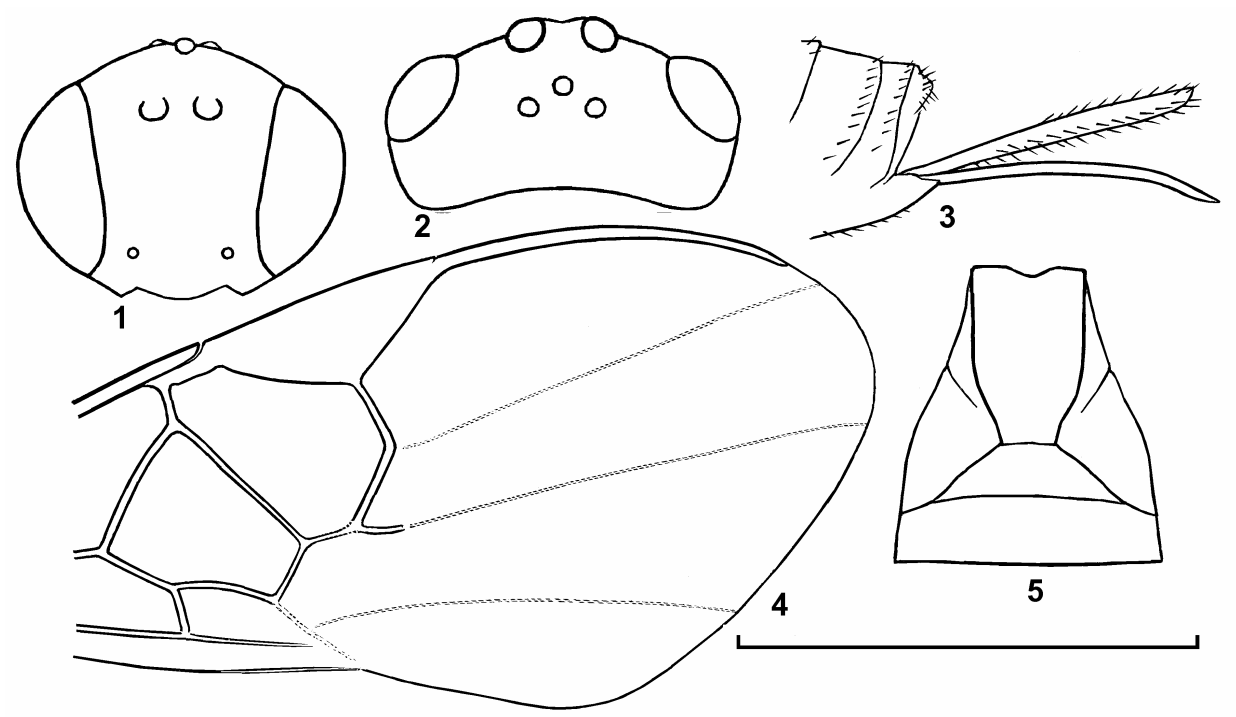

Figs. 1-5. Apanteles derivatus Long, sp. n.

1. Head in frontal view; 2 . Head in dorsal view; 3 . Ovipositor;

4. Fore wing and 5. Metasomal tergites $1+2+3$. 
Mesosoma: in dorsal view width of mesoscutum 0.92 times width of head; mesoscutum sparsely punctate; scutellum and propodeum smooth.

Wings: fore wing 1.2 times as long as body; length of pterostigma 0.8 times metacarp, which 10.4 times as long as its distance from it to apex of marginal cell (fig. 4); parastigma 1.3 times vein 1-SR; vein 1-CU1 0.9 times vein 2-CU1; discal cell as high as wide. Hind wing rather broad, length of cubitellan cell 1.8 times its width.

Legs: Hind tibia 1.9 times basitarsus; inner hind spur 0.65 times as long as hind basitarsus; telotarsus as long as fourth hind tarsus.

Metasoma: first metasomal tergite distinctly narrowed behind middle to apex (fig. 5); length of the first tergite 3.3 times apical width (13:4); first tergite smooth; apical width of median field of second tergite 4.0 times its median length; second tergite 1.3 times as long as third tergite. Ovipositor sheath 0.8 times hind tibia; ovipositor evenly curved throughout (fig. 3).

Colour: Body black; antenna brown, yellowish brown basally; scapus yellow; fore and middle legs yellow; hind coxa reddish brown; hind trochanter, trochantellus and femur yellow; hind tibia and tarsus pale brown, except basal third whitish yellow; wing brownish yellow; first metasomal tergite yellow; 2nd-5th tergites yellowish brown.

Male: unknown.

Remarks: this species is close to $A$. flavigaster $\mathrm{sp} . \mathrm{n}$., but differs by having 1) ocelli in high triangle; 2) first metasomal tergite distinctly narrowed apically and 3) mesoscutum sparsely punctate (densely punctate in flavigaster). The new species is also close to $A$. flavicentrus sp. n. but differs by having: 1), ocelli in high triangle; 2) occiput slightly concave; 3) hind femur yellow.

Etymology: derivativus, Latin for arising from, derive.

Apanteles flavicentrus Long, $\mathrm{sp} . \mathrm{n}$. (figs 6-10).

Material: Holotype, + , Apan. 749, (IEBR), Vietnam: Ha Tinh, Huong Son, $18^{\circ} 22^{\prime} \mathrm{N}$, 106ํํㄹ'E, 900 m, April 20-28, 1998, Malaise, AMNH, K. Long; paratypes, 2q, (IEBR), Mic.161, id. but $1250 \mathrm{~m}$, April 28 1998, as Apan.746, id. but May 18, 1998.

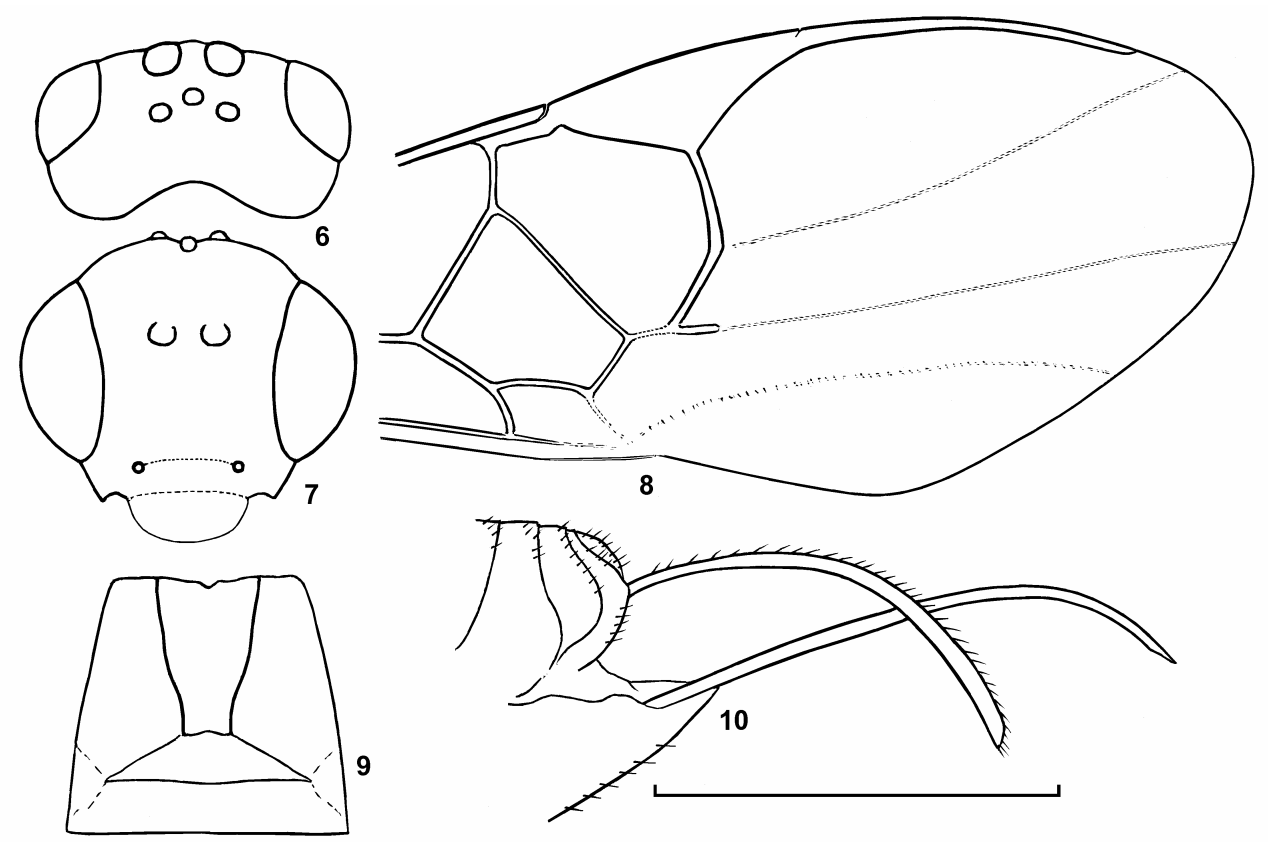

Figs. 6-10. Apanteles flavicentrus Long, sp. n.

6. Head in dorsal view; 7. Head in frontal view; 8 . Fore wing;

9. Metasomal tergites $1+2+3$ and 10 . Ovipositor. 
Holotype: $\circ$, length of body $2.7 \mathrm{~mm}$, of fore wing $3.1 \mathrm{~mm}$, of setose part of ovipositor sheath $1.1 \mathrm{~mm}$ and of antenna $2.6 \mathrm{~mm}$.

Head: antenna slightly shorter body; eyes convergent below (fig. 7); width of face 0.8 times height of eye and subequal median length of face and clypeus combined; distance between tentorial pits 2.25 times distance from pit to eye margin; in dorsal view width of head 2.3 times its median length; occiput deeply concave (fig. 6 ); ocelli in very low triangle, anterior tangent of posterior ocelli cutting anterior ocellus (fig.); distance between posterior ocelli (POL) 1.7 times diameter of posterior ocellus (OD) and 0.8 times distance from posterior ocellus to eye margin (OOL) (fig. 6). Face, frons, vertex and temple densely finely punctate.

Mesosoma: in dorsal view, mesoscutum 1.2 times as wide as width of head (36:30); mesoscutum densely punctate; scutellum shiny with sparse punctures; propodeum smooth.

Wings: fore wing with long pterostigma, 0.9 times as long as metacarp, metacarp 6.0 times as long as its distance from it to apex of the marginal cell (fig. 8); vein $1-\mathrm{CU} 1$ as long as vein 2-CU1. Hind wing broad, length of cubitellan cell 1.25 times its width.

Legs: hind tibia 2.05 times hind basitarsus; inner hind spur 0.5 times as long as hind basitarsus; telotarsus 1.2 times as long as fourth tarsus.

Metasoma: first metasomal tergite narrowed behind middle to apex (fig. 9); length of the first tergite 2.4 times apical width; first tergite smooth; median field of second tergite transverse, its apical width 4.0 times its median length; second tergite 1.2 times third tergite. Ovipositor sheath longer hind tibia (43:39); ovipositor evenly curved throughout (fig. 10).

Colour: body black, antenna brown; scapus yellow; palpi whitish yellow; fore and middle legs yellow, except brown coxa; hind coxa, femur and apical third of tibia brown; hind trochanter, trochantellus and tarsus yellow; first and second metasomal tergites yellow, whitish yellow ventrally; remainder of metasoma brown.

Male: unknown.

Remarks: this species is close to A. prusias from Ceylon, but differs by having: 1). Hind wing rather broad with cubitellan cell wider than high; 2). propodeum smooth; 3). ovipositor sheath distinctly longer hind tibia.

Etymology: flavo, Latin for yellow, first and second metasomal tergites yellow.

Apanteles flavigaster Long, sp. n. (Figs 11-15)

Material: Holotype, + , Apan.984, (IEBR), N.E. Vietnam: Vinh Phuc, Tam Dao, 200m, bushes, 05.IX.2008, K. D. Long; paratypes, 5, (IEBR), Apan.297, C. Vietnam: Quang Tri, Cam Le, Tan Lam, peper darden, 02.VIII.1996, Long; Apan.693, VN: Bac Kan, Ba Be NP, secondary forest 17.VII.2004, K. Long; Apan.792, N.E. Vietnam: Vinh Phuc, Me Linh, fruit orchard, MT, 01-25.XII.2000, K. D. Long; Apan.793, id. but 15-25.X.2000; Apan.1067, N.E. Vietnam: Phu Tho, Xuan Son, Xuan Dai, MT, 1015.VII.2009, K.D.Long, N.H. Thao.

Holotype: 9 , length of body $2.5 \mathrm{~mm}$, of fore wing $2.8 \mathrm{~mm}$, of setose part of ovipositor sheath $0.6 \mathrm{~mm}$ and of antenna $2.5 \mathrm{~mm}$.

Head: antenna as long as body; eyes convergent below (fig. 11); width of face 0.9 times height of eye and 0.8 times median length of face and clypeus combined; distance between tentorial pits 2.0 times distance from pit to eye margin; in dorsal view, width of head 2.1 times median length; ocelli in very low triangle, anterior tangent of posterior ocelli cutting anterior ocellus; distance between posterior ocelli (POL) 2.0 times diameter of posterior ocellus (OD) and 0.8 times distance from posterior ocellus to eye margin (OOL) (fig. 12); face largely punctate, frons, vertex and temple punctate.

Mesosoma: in dorsal view, width of mesoscutum 1.1 times width of head; mesoscutum densely punctate; scutellum narrowed apically and smooth; propodeum shiny, smooth.

Wings: pterostigma 0.7 times as long as metacarp, metacarp 7.5 times as long as its distance from it to apex of marginal cell (Fig. 15); parastigma 1.3 times vein 1-SR; first discal cell higher than wide (18:16); vein 1-CU1 0.9 times vein 2-CU1. Hind wing broad, length of cubitellan cell 2.0 times its width.

Legs: hind tibia 2.1 times hind basitarsus; 
inner hind spur 0.6 times as long as hind basitarsus; telotarsus 1.3 times fourth segment.

Metasoma: first metasomal tergite slightly narrowed at apex (fig. 14); length of the first tergite 1.7 times its apical width; first tergite smooth; apical width of median field of second tergite 4.0 times its median length; second metasomal tergite 1.5 times third tergite; ovipositor sheath 0.9 times as long as hind tibia; ovipositor evenly curved throughout (fig. 13).

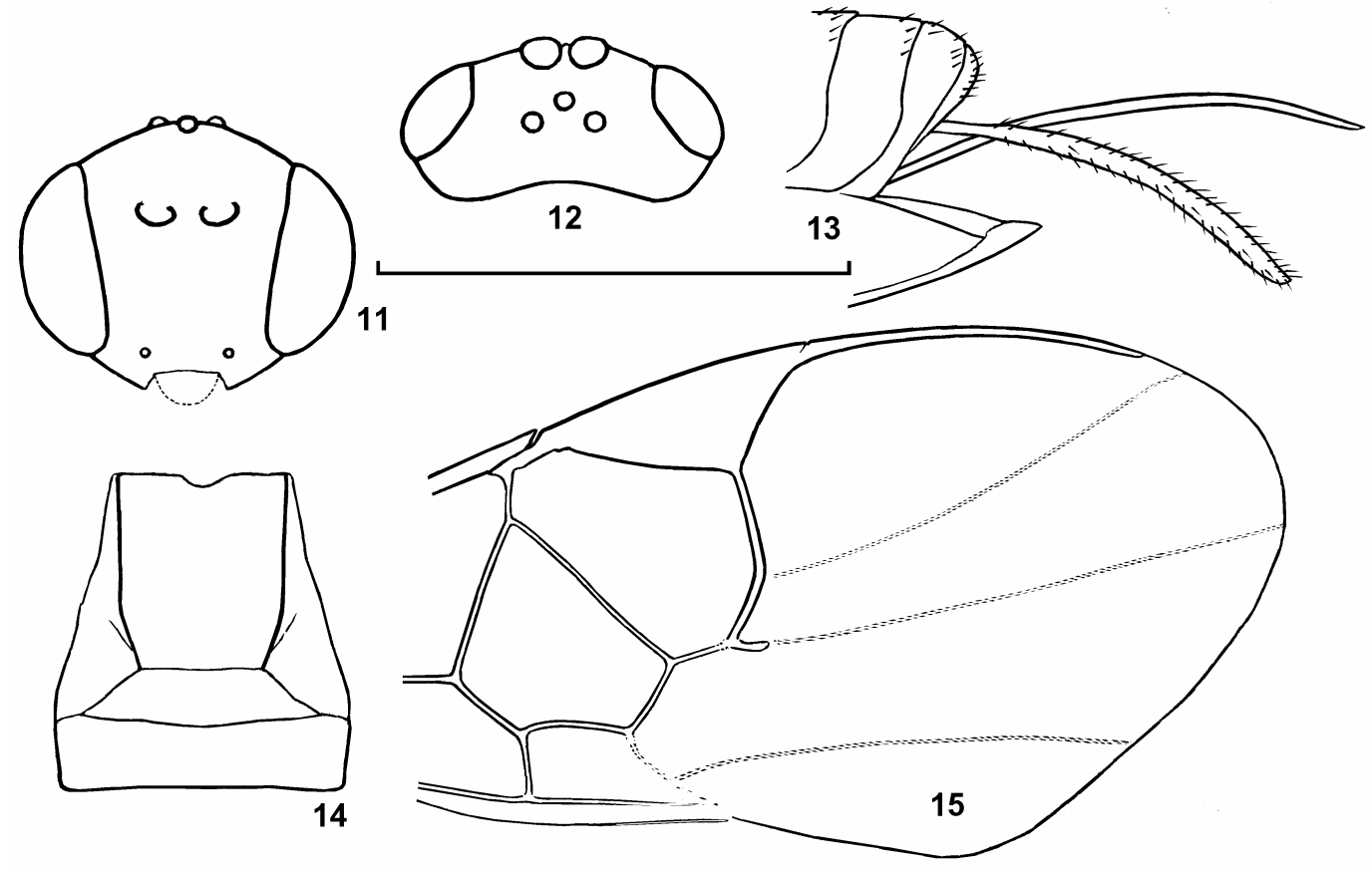

Figs 11-15. Apanteles flavigaster Long, sp. $\mathrm{n}$.

11. Head in frontal view; 12. Head in dorsal view; 13. Ovipositor;

14. Metasomal tergites $1+2+3$ and 15 . Fore wing.

Colour: Head and mesosoma black; metasoma yellow; scapus yellow; flagellum brown apically, yellowish brown basally; palpi yellow; fore and middle legs yellow, except coxa brown; hind leg yellow, except coxa, apical half of tibia brown.

Male: unknown

Remarks: This species is close to Apanteles flavicentrus sp. n., but differs by having: 1) occiput slightly concave; 2). face largely punctate; 3). first metasomal tergite slightly narrowed at apex; 4). ovipositor sheath shorter hind tibia.

Etymology: Flavo, Latin for yellow, and gaster for metasoma.

Apanteles hatinhensis Long, sp. n. (Figs 16-20)

Material: Holotype, $q$, Mic. 114, (IEBR), C. Vietnam: Ha Tinh, Huong Son, $18^{\circ} 22^{\prime} \mathrm{N}$,
106¹3’E, 600 m, April 22-May 1, 1998, Malaise, AMNH, K. Long; paratype, 1웅 (IEBR), Apan.1064, N.E. Vietnam: Vinh Phuc, Me Linh, Ngoc Thanh, fruit orchard, MT, 726.VI.2001, K. D. Long.

Holotype: 9 , length of body $2.8 \mathrm{~mm}$, of fore wing $3.2 \mathrm{~mm}$, of setose part of ovipositor sheath $1.5 \mathrm{~mm}$ and of antenna $3.0 \mathrm{~mm}$.

Head: Antenna longer than body; eyes not convergent below (fig. 17); eye rather large, width of face 0.8 times height of eye and 0.9 times median length of face and clypeus combined; distance between tentorial pits 3.0 times distance from pit to eye margin; head transverse, in dorsal view width of head 2.5 median length; ocelli in low triangle, anterior tangent of posterior ocelli touching posterior margin of anterior ocellus; distance between posterior ocelli (POL) equal to diameter of posterior ocellus (OD) and 0.6 times 
distance from posterior ocellus to eye margin (OOL) (Fig. 16); face, frons, vertex and temple finely punctate.

Mesosoma: in dorsal view, width of mesoscutum 0.9 times width of head; mesoscutum densely and finely punctate; scutellum smooth with fine punctures laterally; propodeum shiny with sparse fine punctures.

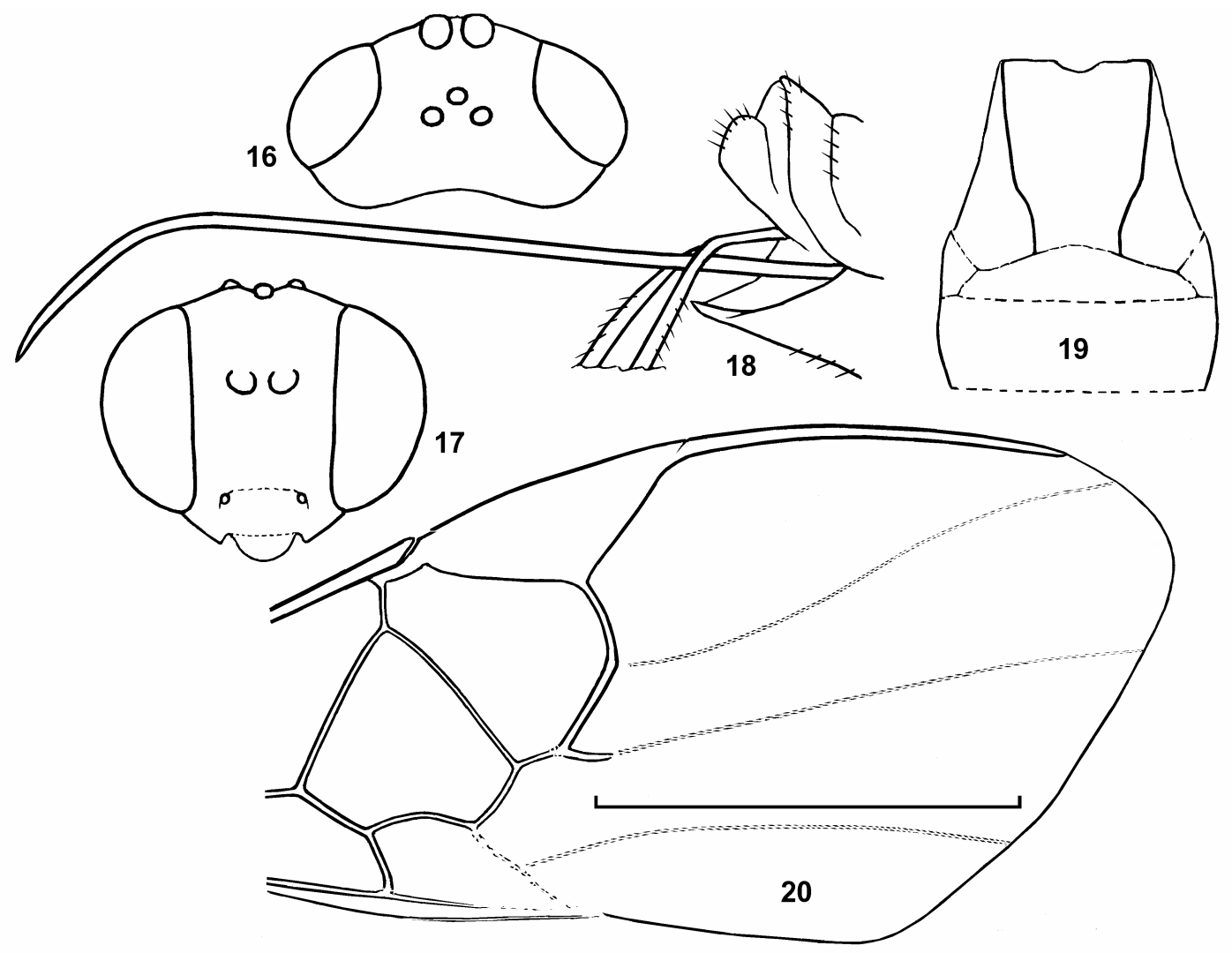

Figs. 16-20. Apanteles hatinhensis Long, sp. n.

16. Head in dorsal view; 17 . Head in frontal view; 18 . Ovipositor;

19. Metasomal tergites $1+2+3$ and 20 . Fore wing.

Wings: pterostigma 0.8 times metacarp, metacarp 6.4 times as long as its distance from metacarp to apex of the marginal cell (fig. 20); parastigma as long as vein 1-SR; first discal cell as high as wide; vein 1 -CU1 0.8 vein 2 -CU1. Hind wing missing in holotype, in paratype length of cubitellan cell 1.6 times its width.

Legs: hind tibia missing in holotype, but in paratype hind tibia 2.3 times hind basitarsus; inner hind spur 0.5 times as long as hind basitarsus; telotarsus 1.3 times fourth segment.

Metasoma: first metasomal tergite distinctly narrowed behind middle to apex (fig. 19); length of the first tergite 2.3 times its apical width; first tergite smooth, rugose apically; median field of second tergite transverse, its apical width 4.0 times its median length; second metasomal tergite 0.6 times as long as third tergite; ovipositor sheath very long, 1.7 times hind tibia; ovipositor straight basally, abruptly curved down apically (fig. 18).

Colour: body black, scapus yellow; flagellum brown; palpi whitish yellow; wings brown; fore and middle legs yellow; hind leg yellow, except hind coxa brown basally.

Male: unknown.

Remarks: this species is close to A. hebrus Nixon, 1965, from Africa, but differs by having: 1). POL shorter than OOL; 2). propodeum shiny and smooth; 3). hind inner spur 0.5 times as long as hind basitarsus (longer in A. hebrus); 4). ovipositor sheath very long, much longer than hind tibia (ovipositor sheath shorter than hind tibia in hebrus). 
Etymology: after Ha Tinh, the locality where type was collected.

Apanteles paraglaope Long, sp. n. (figs 21-26)

Material: Holotype, , Apan. 796, (IEBR), N.E. Vietnam: Vinh Phuc, Me Linh, Ngoc Thanh, fruit orchard, MT, 01-25.XII.2000, K. D. Long.

Holotype: , , length of body $2.5 \mathrm{~mm}$, of fore wing $2.8 \mathrm{~mm}$, of setose part of ovipositor sheath $1.1 \mathrm{~mm}$ and of antenna $2.2 \mathrm{~mm}$.

Head: antenna shorter than body; eyes slightly convergent below (fig. 21); width of face 1.1 times height of eye and as long as median length of face and clypeus combined; distance between tentorial pits 2.7 times distance from pit to eye margin; in dorsal view, width of head 1.9 times its median length; ocelli in rather low triangle, anterior tangent of posterior ocelli touching posterior margin of anterior ocellus; distance between posterior ocelli (POL) 2.5 times diameter of posterior ocellus (OD) and equal to distance from posterior ocellus to eye margin (OOL); temple almost perpendicularly and slightly narrowed posteriorly (fig. 22); face, frons, vertex and temple densely and finely punctate.

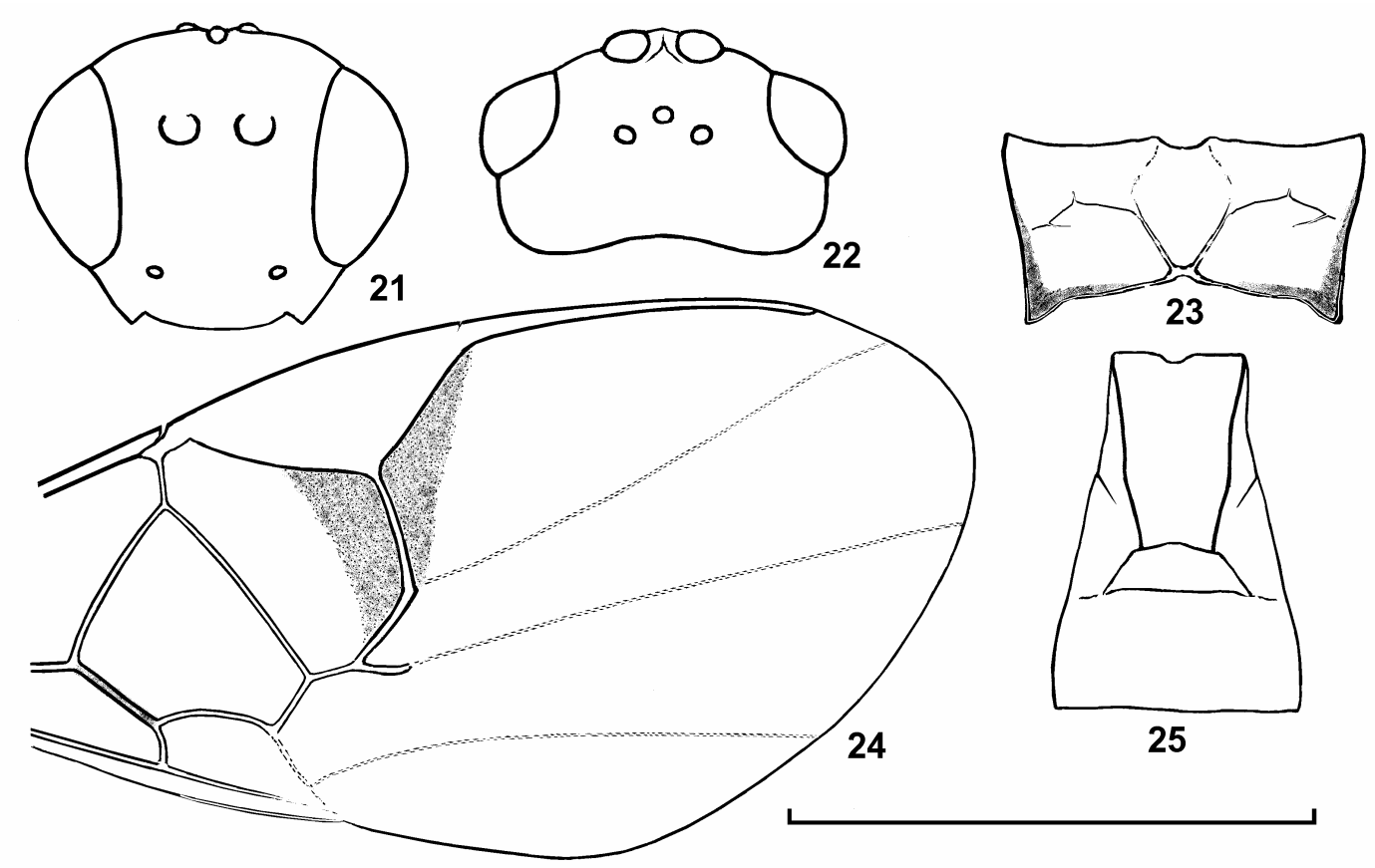

Figs. 21-25. Apanteles paraglaope Long, sp. $\mathrm{n}$.

21. Head in dorsal view; 22. Head in frontal view; 23. Propodeum;

24. Fore wing and 25 . Metasomal tergites $1+2+3$.

Mesosoma: in dorsal view, width of mesoscutum width of head; mesoscutum densely punctate anteriorly, rugose-punctate medio-apically; scutellum smooth; propodeum with an areola and costulae (fig. 23).

Wings: vein $r 1.6$ times as long as vein 2-SR (fig. 24); pterostigma broad, 0.9 times as long as metacarp; metacarp 4.8 times distance from it to apex of marginal cell; hind wing pallid.

Legs: hind tibia 2.0 times hind basitarsus; inner hind spur 0.5 times as long as hind basitarsus; hind telotarsus 1.3 times as long as fourth segment.

Metasoma: first metasomal tergite distinctly narrowed apically (fig. 25); length of the first tergite 2.6 times its apical width; apical width of median field of second tergite 3.0 times its median length; second tergite 0.4 times as long as third tergite; ovipositor sheath 1.5 times longer than hind tibia; ovipositor nearly straight, hypopygium developed; first tergite rugose, $2^{\text {nd }}$ +3 rd tergites smooth. 
Colour: body black; antenna dark brown; fore wing with dark brown cloud beneath pterostigma, veins $\mathrm{r}, 2-\mathrm{SR}$ and 1-CU dark brown (fig. 24); fore and middle blackish brown, except for fore femur apically, fore and middle tibia ivory basally; hind leg black except basal one third of tibia and spurs whitish yellow.

Male: unknown.

Remarks: this species is close to A. aglaope from Indonesia (Sumatra), but differs by having: 1). Ovipositor sheath much longer than hind tibia; 2). Ovipositor thin and without apical constriction; 3). Median field of second metasomal tergite less transverse.

Etymology: para, Greek for beside, near and the specific name aglaope.

Acknowledgements: This paper was supported by National Foundation for Science and Technology Development (NAFOSTED), Vietnam (grant 106.15.04.09). I express thanks to Dr. Cornelis van Achterberg from the Netherlands Centre for Biodiversity Naturalis, Leiden (the Netherlands) for comments and correction of the English text.

\section{REFERENCES}

1. Achterberg van C., 1993: Illustrated key to the subfamilies of the Braconidae (Hymenoptera: Ichneumonoidea). Zool. Verh. Leiden 283: 1-189.

2. Austin A. D., Dangerfield P. C., 1992: Invertebrate Taxonomy, 6(1): 1-76.

3. Khuat Dang Long, 2007: Journal of Biology, 29(3): 25-31. Vietnam.

4. Khuat dang Long, Belokobylskij S. A., 2003: Russian Entomological Journal, 12(4): 385-398.

5. Kotenko A. G., 1981: Vesnik Zoologii, 2: 26-30.

6. Mason W. R. M., 1981: Mem. Entom. Soc. Canada, 115: 1-147.

7. Nixon G. E. J., 1965: Bull. Br. Mus. nat. Hist. Entomology, Suppl., 2: 1-284.

8. Yu D. S., Achterberg van C., Horstmann K., 2005: Ichneumonoidea 2004 (Biological and taxonomical information), Taxapad Interactive Catalogue, Vancouver.

\title{
NĂM LOÀI MỚI CHO KHOA HỌC THUỘC GIỐNG APANTELES FOERSTER (HYMENOPTERA: BRACONIDAE: MICROGASTRINAE) Ở VIẸT NAM
}

\author{
KHUẤT ĐĂNG LONG
}

\begin{abstract}
TÓM TÁ́T
Giống Apanteles Foerster, 1862 gồm các loài ong ký sinh có tên chung là ong kén trắng, hầu hết chúng là loài ong ký sinh ở pha sâu non của nhiều loài côn trùng khác (chủ yếu ở các loài sâu hại thuộc bộ cánh Vảy (Lepidoptera). Do số lượng loài rất lớn tập trung trong một giống, chúng được Nixon (1965) [7] chia ra các nhóm loài, sau đó trên cơ sở nghiên cứu phát sinh loài, Mason (1981) đã tách một số nhóm thành giống độc lập, nhiều nhóm trong số đó đã được công nhận là tên giống chính thức [8], những nhóm khác còn lại vẫn thuộc giống Apanteles.

Trong một công tình trước đây [3], chúng tôi đã mô tả 2 loài mới cho khoa học thuộc nhóm Apantelesmerula, trong bài này chúng tôi mô tả 3 loài mới cũng thuộc nhóm này và 1 loài mới thuộc nhóm Apantelesater. Khóa định loại cũng được thiết lập cho 5 loài thuộc nhóm Apanteles - merula ở Việt Nam.
\end{abstract}

\section{Nhóm Apanteles MERULA Nixon, 1965}

Đặc điểm chẩn loại: Đốt trung gian (propodeum) không có các khoang lõm với gờ viền, thường nhẵn; rìa viền dưới phần gốc cánh sau lõm rõ, nhẵn không có lông măng; cánh trước có gân $r$ và gân 2-SR ít tạo góc gãy 
rõ (hình $4,8,15$ ); hai rìa bên phía gốc tấm lưng bụng 1 song song sau đó hẹp đột ngột về phía đỉnh (hình 5,9 ); máng đẻ trứng ít nhất thường dài bằng $3 / 4$ ống chân sau. Khóa định loại cho 21 loài thuộc nhóm Apanteles merula, trong số đó có 9 loài của khu hệ Đông Phương - Ấn Độ tham khảo trong công trình của Nixon (1965) [7]. Khóa định loại cho 12 loài thuộc nhóm Apanteles-merula của khu hệ châu Âu tham khảo trong công trình của Kotenlo (1981) [5].

Nixon (1965) [7] đã đưa ra khóa định loại cho 21 loài thuộc nhóm này, trong số đó có 9 loài thuộc khu hệ Indo - Australian, 7 loài thuộc châu Âu và 5 loài thuộc châu Phi. Khuất Đăng Long (2007) [3] đã mồ tả 2 loài thuộc nhóm này là A. gialamensis Long K, 2007 và A. subcamilla Long K, 2007.

\section{Khóa định loại 5 loài thuộc nhóm Apanteles-merula ở Việt Nam}

1. Ba mắt đơn nằm ở vị trí khá cao, tiếp tuyến trước của hai mắt đơn sau không cắt gờ sau của mắt đơn trước (hình 2)

- Ba mắt đơn nằm ở vị trí thấp, tiếp tuyến trước của hai mắt đơn sau cắt vào mắt đơn trước (hình 6,2 và 16 ).

2. Mắt cánh trong suốt với đường viền xẫm màu (xem hình 1, Khuat Dang Long, 2007: 26); máng đẻ trứng gần như thẳng chỉ cong gập xuống ở sát đỉnh (xem hình 7, Khuat Dang Long, 2007: 26)....................................................... gialamensis Long K, 2007

- Mắt cánh nâu (hình); máng đẻ trứng cong đều từ gốc đến đỉnh (hình 3) A. derivatus sp. $\mathrm{n}$.

3. Tấm lưng bụng 1 chỉ hơi hẹp ở sát đỉnh (hình 12, 14, Khuất Đăng Long, 2007: 28).. 4

- Tấm lưng bụng 1 hẹp rõ từ giữa đến đỉnh (hình 9,19$)$ )...........................................................................

4. Gân sau mắt cánh khá ngắn, chỉ dài gấp 3,0 lần khoảng cách từ gân này đến đỉnh ô cánh radial; mắt cánh trong suốt với đường viền đậm màu (hình13, Khuat Dang Long, 2007: 28); bụng màu đen. A. subcamilla Long K, 2007

- Gân sau mắt cánh rất dài, gấp 7,5 lần khoảng cách từ gân này đến đỉnh ô cánh radial (hình 15); mắt cánh nâu toàn bộ; bụng màu vàng... A. flavigaster $\mathrm{sp} . \mathrm{n}$.

5. Rìa trong hai mắt kép chụm xuống phía dưới (hình 7); đùi sau màu nâu tối; bao máng đẻ trứng dài hơn ít so với ống chân sau; máng đẻ trứng cong đều từ gốc đến đỉnh (hình 10). A. flavicentrus sp. $\mathrm{n}$.

- Rìa trong hai mắt kép thẳng phía dưới (hình 17); đùi sau màu vàng; bao máng đẻ trứng dài hơn 1,5 lần ống chân sau; máng đẻ trứng gần như thẳng, chỉ cong gập đột ngột ở sát đỉnh (hình 18). A. hatinhensis sp. $\mathrm{n}$.

Apanteles gialamensis Long K, 2007

Tạp chí Sinh học, 2007, 29(3): 25 (hình 1-7).

Mẫu kiểm tra: Holotyp, + , Apan.508, (IEBR), Việt Nam: Hà Nội (Gia Lâm), thu trên ruộng đậu đỗ, ngô, 13.IV.2006, K. D. Long; paratyp: 2 ? Apan.508a, Apan.508b, 2ð : Apan.508c, Apan.508d, (IEBR): địa điểm như holotyp; 1 + Apan.634, (IEBR); VN, Hà Nội (Gia Lâm), 20.V.2006, K.D. Long.

\section{Apanteles subcamilla Long K, 2007}

Tạp chí Sinh học, 2007, 29(3): 27 (hình 8-13).

Mẫu kiểm tra: Holotyp, + , Apan.036, (IEBR), VN: Hà Nội (Gia Lâm), trong vườn, bẫy màn treo (MT), 205'N 105'55'E, 15-25.XI.2001, K. D. Long; paratyp: 3 + Apan.037, Apan.038, Apan.041, (IEBR), địa điểm như ở holotyp, MT 25.XI-05.XII.2001; 2 Apan.685, Apan.686, (IEBR); VN, Vĩnh Phúc (Mê Linh), MT, 11-25.X.2006, K.D. Long.

\section{Apanteles derivatus Long, sp. n. (hình 1-5)}

Mẫu vật: Holotype, †, Apan.863, (IEBR), Tây Bắc Việt Nam: Lào Cai, Nam Cường, rừng TS, 11.VI.2004, K. D. Long.

Holotype: + , thân dài $2,2 \mathrm{~mm}$, cánh trước dài $2,6 \mathrm{~mm}$, phần phủ lông măng bao máng đẻ trứng dài 0,7 $\mathrm{mm}$ và râu dài $2,3 \mathrm{~mm}$. 


\section{Con đực: Chưa rõ.}

Nhận xét: Loài này gần với $A$. flavogaster sp. n., nhưng có đặc điểm khác ở chỗ: 1 . ba mắt đơn cao, tiếp tuyến rìa trước của hai mắt đơn sau không cắt rìa sau của mắt đơn trước; 2 . mesoscutum có chấm lỗ thưa; 3 . tấm lưng bụng 1 hẹp rõ dần từ giữa đến đỉnh. Loài này cũng gần với loài $A$. flavogaster sp. n., nhưng khác ở chỗ: 1 . ba mắt đơn cao, tiếp tuyến rìa trước của hai mắt đơn sau không cắt rìa sau của mắt đơn trước; 2 . gáy hơi lõm vào; 3 . đùi sau màu vàng.

Apanteles flavicentrus Long, sp. n. (hình 6-10)

Mẫu vật: Holotyp, + , Apan.749, (IEBR), Việt Nam: Hà Tĩnh, Hương Sơn, $18^{\circ} 22^{\prime} \mathrm{N}, 106^{\circ} 13^{\prime} \mathrm{E}, 900$ m, April 20-28, 1998, Malaise, AMNH, K. Long; paratyp, 2+, (IEBR), Mic.161, giống như trước nhưng ở 1250 m, April 28 1998, như Apan.746, May 18, 1998.

Holotype: + , thân dài $2,7 \mathrm{~mm}$, cánh trước dài $3,1 \mathrm{~mm}$, phần phủ lông măng bao máng đẻ trứng dài 1,1 $\mathrm{mm}$ và râu dài $2,6 \mathrm{~mm}$.

Con đực: Chưa rõ.

Nhận xét: Loài này gần với $A$. prusias Nixon, 1965 của Sri Lanca, nhưng có đặc điểm khác ở chỗ: 1. cánh sau rộng có ô cánh cubitellan rộng hơn chiều cao; 2- đốt trung gian nhẵn; 3. bao máng đẻ trứng dài hơn ống chân sau rất rõ.

Apanteles flavigaster Long, sp. n. (hình 11-15)

Mẫu vật: Holotyp, +, Apan.984, (IEBR), Đông Bắc Việt Nam: Vĩnh Phúc, Tam Đảo, 200 m, cây bụi, 05.IX.2008, K. D. Long; paratyp, 4오, (IEBR), Apan.297, Trung bộ Việt Nam: Quảng Trị, Cẩm Lệ, Tân Lâm, vườn tiêu, 02.VIII.1996, Long; Apan.693, VN: Bắc Kạn, VQG Ba Bể, rừng TS 17.VII.2004, K. Long; Apan.792, Đông Bắc Việt Nam: Vĩnh Phúc, Mê Linh, vườn quả, MT, 01-25.XII.2000, K. D. Long; Apan.793, địa điểm như trên nhưng 15-25.X.2000; Apan.1067, Apan.1067, Đông Bắc Việt Nam: Phú Thọ, Xuân Sơn, Xuân Đài, MT, 10-15.VII.2009, K.D.Long, N.H. Thảo.

Holotype: 9 , thân dài $2,5 \mathrm{~mm}$, cánh trước dài $2,8 \mathrm{~mm}$, phần phủ lông măng bao máng đẻ trứng dài 0,6 $\mathrm{mm}$ và râu dài $2,5 \mathrm{~mm}$.

Con đực: Chưa rõ.

Nhận xét: Loài này gần với $A$. flavicentrus sp. n., nhưng có đặc điểm khác ở chỗ: 1 . gáy hơi lõm vào; 2. mặt có chấm lõm thô và dày; 3 . tấm lưng bụng 1 chỉ hơi hẹp sát đỉnh; 4 . bao máng đẻ trứng ngắn hơn ống chân sau.

\section{Apanteles hatinhensis Long, sp. n. (hình 16-20)}

Mẫu vật: Holotyp, + , Mic.114, (IEBR), Bắc Trung bộ Việt Nam: Hà Tĩnh, Hương Sơn, 18²2'N, 106 ${ }^{\circ} 13^{\prime} \mathrm{E}, 600$ m, April 22-May 1, 1998, Malaise, AMNH, K. Long; paratyp, 1우, (IEBR), Apan.1064, Đông Bắc Việt Nam: Vĩnh Phúc, Mê Linh, Ngọc Thanh, vườn quả, MT, 7-26.VI.2001, K. D. Long.

Holotype: + , thân dài $2,8 \mathrm{~mm}$, cánh trước dài $3,2 \mathrm{~mm}$, phần phủ lông măng bao máng đẻ trứng dài 1,5 $\mathrm{mm}$ và râu dài $3,0 \mathrm{~mm}$.

Con đực: Chưa rõ.

Nhận xét: Loài này gần với $A$. hebrus Nixon, 1965 của châu Phi, nhưng có đặc điểm khác ở chỗ: 1. khoảng cách giữa hai mắt đơn sau ngắn hơn khoảng cách từ mắt đơn sau đến rìa mắt kép; 2 . đốt trung gian nhẵn bóng; 3 . cựa trong ống chân sau dài bằng 0,5 lần đốt bàn 1 chân sau; 4 . bao máng đẻ trứng rất dài (dài bằng 1,5 lần ống chân sau).

\section{Nhóm Apanteles ATER Nixon, 1965}

Đặc điểm chẩn loại: Đốt trung gian (propodeum) có các khoang lõm với gờ viền, hoặc ít nhất có có gờ nhăn; rìa viền dưới phần gốc cánh sau lõm rõ, nhẵn không có lông măng; cánh trước có các đám nâu xẫm màu ở dưới mắt cánh có gân $\mathrm{r}$ (hình); máng đẻ trứng ít nhất thường dài bằng $3 / 4$ ống chân sau. Đây là một nhóm loài có số lượng rất lớn, tuy nhiên mới gặp một loài, Apanteles aglaope Nixon, 1965 của Inđônêxia (Sumatra) (Nixon, 1965) [7], có đám nâu xẫm dưới mắt cánh. Khóa định loại 125 loài thuộc nhóm Apanteles-ater của khu hệ Đông Phương-ấn Độ tham khảo trong công trình của Nixon (1965). 
Apanteles paraglaope Long, sp. n. (hình 21-25)

Mẫu vật: Holotyp, †, Apan.796, (IEBR), Đông Bắc Việt Nam: Vĩnh Phúc, Mê Linh, Ngọc Thanh, vườn quả, MT, 01-25.xii.2000, K. D. Long.

Holotype: , thân dài $2,5 \mathrm{~mm}$, cánh trước dài $2,8 \mathrm{~mm}$, phần phủ lông măng bao máng đẻ trứng dài 1,1 mm và râu dài $2,2 \mathrm{~mm}$.

Con đực: Chưa rõ.

Nhận xét: Loài này gần với loài Apanteles aglaope Nixon, 1965 của Inđônêxia (Sumatra), nhưng có đặc điểm khác ở chỗ: 1. bao máng đẻ trứng dài hơn ống chân sau; 2. máng đẻ trứng mảnh hơn; 3. quầng ở gốc tấm lưng bụng 2 ngắn và ít dẹt hơn (hình 25 ).

Ngày nhận bài: 15-6-2010 